MALAYA JOURNAL OF MATEMATIK

Malaya J. Mat. 09(02)(2021), 12-19.

http://doi.org/10.26637/mjm0902/002

\title{
Approximating positive solutions of nonlinear IVPs of ordinary second order hybrid differential equations
}

\author{
Bapurao C. Dhage ${ }^{* 1}$ Janhavi B. Dhage ${ }^{2}$ \\ 1 “Kasubai”, Gurukul Colony, Thodga Road, Ahmedpur-413 515, Dist. Latur, Maharashtra, India. \\ 2 “Kasubai”, Gurukul Colony, Thodga Road, Ahmedpur-413 515, Dist. Latur, Maharashtra, India.
}

Received 12 February 2021; Accepted 28 March 2021

\begin{abstract}
In this paper we prove the existence and approximation of solution for a nonlinear initial value problem of ordinary second order hybrid differential equation. The right hand side of the differential equation is assumed to be Carathoèodory and the proof is based on a Dhage iteration method.
\end{abstract}

AMS Subject Classifications: 34A45, 34B15, 47H07, 47H10.

Keywords: Nonlinear initial value problems, Hybrid differential equation, Dhage iteration method, Existence and approximation theorem.

\section{Contents}

1 Introduction and Background $\quad 12$

2 Auxiliary Results $\quad 13$

3 Existence and Approximation Result $\quad 14$

4 An Example $\quad 17$

\section{Introduction and Background}

Let $\mathbb{R}$ denote the set of all real numbers and $\mathbb{R}_{+}$the set of all nonnegative reals. Given a closed and bounded interval $J=[0, T] \subset \mathbb{R}$, consider the nonlinear hybrid initial value problem (in short HIVP) of ordinary second order hybrid differential equation (in short HDE),

$$
\left.\begin{array}{c}
\frac{d^{2}}{d t^{2}}\left(\frac{x(t)}{f(t, x(t))}\right)=g(t, x(t)) \text { a.e. } t \in J, \\
x(0)=0, \quad x^{\prime}(0)=0,
\end{array}\right\}
$$

where $f: J \times \mathbb{R} \rightarrow \mathbb{R} \backslash\{0\}$ is continuous and $f: J \times \mathbb{R} \rightarrow \mathbb{R}$ is a Carathèodory function.

When $f \equiv 1$ on $J \times \mathbb{R}$, the HIVP (1.1) reduces to the well-known nonlinear ordinary second order differential equation

$$
\left.\begin{array}{c}
x^{\prime \prime}(t)=g(t, x(t)) \text { a.e. } t \in J, \\
x(0)=0, x^{\prime}(0)=0,
\end{array}\right\}
$$

which is studied earlier extensively in the literature (see Dhage and Dhage [5]).

*Corresponding author. Email address: bcdhage@gmail.com (Bapurao C. Dhage) 
Approximation results for nonlinear hybrid boundary value problems

Definition 1.1. A function $x \in A C^{1}(J, \mathbb{R})$ is said to be a lower solution of the IVP (1.1) if

$$
\left.\begin{array}{c}
\frac{d^{2}}{d t^{2}}\left(\frac{x(t)}{f(t, x(t))}\right) \leq g(t, x(t)) \text { a.e. } t \in J, \\
x(0)=0, x^{\prime}(0)=0,
\end{array}\right\}
$$

where, $A C^{1}(J, \mathbb{R})$ is the space of functions $x \in C(J, \mathbb{R})$ whose first derivative exists and is absolutely continuous on I. Similarly, $x \in A C^{1}(J, \mathbb{R})$ is called an upper solution of (1.1) on $J$ if the reversed inequalities hold in (1.3). If equalities hold in (1.3), we say that $x$ is a solution of (1.1) on J.

The existence of the solution to the problem (1.1) may be proved by using hybrid fixed point theorems of Dhage in a Banach algebra as did in Dhage [2] and Dhage and Imdad [7]. The existence of positive solution to a nonlinear equation is generally proved using the properties of cones in a partially ordered Banach space (see Deimling [1] and Granas [8]). However, the existence and approximation result for the second order IVPs and PBVPs are already proved in Dhage and Dhage $[5,6]$ without using the properties of the cones via a new Dhage iteration method developed in [3]. In the present paper, we shall extend above Dhage iteration method to the HIVP (1.1) and study the existence and approximation of positive solutions of under certain hybrid conditions on the nonlinearities $f$ and $g$ from algebra, analysis and topology.

\section{Auxiliary Results}

We need the following definition in what follows.

Definition 2.1. A function $\beta: J \times \mathbb{R} \rightarrow \mathbb{R}$ is called Carathéodory if

(i) the map $t \mapsto \beta(t, x)$ is measurable for each $x \in \mathbb{R}$, and

(ii) the map $x \mapsto \beta(t, x)$ is continuous for each $t \in J$.

The following lemma is often used in the study of nonlinear differential equations (see Dhage [2] and references therein).

Lemma 2.2 (Carathéodory). Let $\beta: J \times \mathbb{R} \rightarrow \mathbb{R}$ be a Carathéodory function. Then the map $(t, x) \mapsto \beta(t, x)$ is jointly measurable. In particular the map $t \mapsto \beta(t, x(t))$ is measurable on $J$ for each $x \in C(J, \mathbb{R})$.

We need the following hypotheses in the sequel.

$\left(\mathrm{H}_{1}\right) f$ defines a continuous bounded function $f: J \times \mathbb{R} \rightarrow \mathbb{R}_{+} \backslash\{0\}$ with bound $M_{f}$.

$\left(\mathrm{H}_{2}\right)$ There exists a $\mathcal{D}$-function $\varphi_{f} \in \mathfrak{D}$ such that

$$
0 \leq f(t, x)-f(t, y) \leq \varphi_{f}(x-y)
$$

for all $\in J$ and $x, y \in \mathbb{R}$ with $x \geq y$. Moreover, $T^{2} M_{g} \varphi_{f}(r)<r, \quad r>0$.

$\left(\mathrm{H}_{3}\right)$ The function $g$ is Carathéodory on $J \times \mathbb{R}$ into $\mathbb{R}_{+}$.

$\left(\mathrm{H}_{4}\right) g$ is bounded on $J \times \mathbb{R}$ with bound $M_{g}$.

$\left(\mathrm{H}_{5}\right) g(t, x)$ is nondecreasing in $x$ for each $t \in J$.

(LS) The HIVP (1.1) and (1.3) has a lower solution $u \in A C^{1}(J, \mathbb{R})$.

(US) The HIVP (1.1) and (1.3) has an upper solution $v \in A C^{1}(J, \mathbb{R})$. 
Lemma 2.3. Given any function $h \in L^{1}(J, \mathbb{R})$, the HIVP

$$
\left.\begin{array}{c}
\frac{d^{2}}{d t^{2}}\left(\frac{x(t)}{f(t, x(t))}\right)=h(t) \text { a.e. } t \in J, \\
x(0)=0, \quad x^{\prime}(0)=0,
\end{array}\right\}
$$

is equivalent to the quadratic hybrid integral equation (in short HIE)

$$
x(t)=[f(t, x(t))]\left(\int_{0}^{t}(t-s) h(s) d s\right), t \in J .
$$

The proof of our main result will be based on the Dhage monotone iteration principle or Dhage monotone iteration method contained in a applicable hybrid fixed point theorem in the partially ordered Banach algebras.

A non-empty closed convex subset $K$ of the Banach algebra $E$ is called a cone if it satisfies i) $K+K \subset K$, ii) $\lambda K \subseteq K$ for $\lambda>0$ and iii) $\{-K\} \cap K=\{0\}$. We define a partial order $\preceq$ in $E$ by the relation $x \preceq y \Longleftrightarrow$ $y-x \in K$. The cone $K$ is called positive if iv) $K \circ K \subseteq K$, where "o" is a multiplicative composition in $E$. In what follows we assume that the cone $K$ in a partially ordered Banach algebra $(E, K)$ is always positive. Then the following results are known in the literature.

Lemma 2.4 (Dhage [4]). Every ordered Banach space $(E, K)$ is regular.

Lemma 2.5 (Dhage [4]). Every partially compact subset $S$ of an ordered Banach space $(E, K)$ is a Janhavi set in $E$.

Theorem 2.6 (Dhage [3]). Let $(E, K,\|\cdot\|)$ be a regular partially ordered complete normed linear algebra and let every chain $C$ in $E$ be a Janhavi set. Suppose that $\mathcal{A}, \mathcal{B}: E \rightarrow K$ are two monotone nondecreasing operators such that

(a) $\mathcal{A}$ is partially bounded and partial $\mathcal{D}$-Lipschitz with $\mathcal{D}$-function $\varphi_{\mathcal{A}}$,

(b) $\mathcal{B}$ is partially continuous and uniformly partially compact,

(c) $M_{\mathcal{B}} \varphi_{\mathcal{A}}(r)<r, r>0$, where $M_{\mathcal{B}}=\sup \{\|\mathcal{B}(C)\|: C$ is a chain in $E\}$, and

(d) there exists an element $x_{0} \in E$ such that $x_{0} \preceq \mathcal{A} x_{0} \mathcal{B} x_{0}$ or $x_{0} \succeq \mathcal{A} x_{0} \mathcal{B} x_{0}$.

Then the hybrid operator equation $\mathcal{A} x \mathcal{B} x=x$ has a solution $x^{*}$ in $K$ and the sequence $\left\{x_{n}\right\}_{n=0}^{\infty}$ of successive iterations defined by $x_{n+1}=\mathcal{A} x_{n} \mathcal{B} x_{n}$ converges monotonically to $x^{*}$.

The details of Dhage monotone iteration principle or method and related definitions of Janhavi set and uniformly partially compact operator along with some applications may be found in Dhage $[3,4]$ and the references therein.

\section{Existence and Approximation Result}

Let $C_{+}(J, \mathbb{R})$ denote the space of all nonnegative-valued functions of $C(J, \mathbb{R})$. We assume that the space $C(J, \mathbb{R})$ is endowed with the norm $\|\cdot\|$ and the multiplication "." defined by

$$
\|x\|=\max _{t \in J}|x(t)| \quad \text { and }(x \cdot y)(t)=x(t) y(t) t \in J .
$$

We define a partial order $\preceq$ in $E$ with the help of the cone $K$ in $E$ defined by

$$
K=\{x \in E \mid x(t) \geq 0 \text { for all } t \in J\}=C_{+}(J, \mathbb{R}),
$$


Approximation results for nonlinear hybrid boundary value problems

which is obviously a positive cone in $C(J, \mathbb{R})$. Thus, we have $x \preceq y \Longleftrightarrow y-x \in K$.

Clearly, $C(J, \mathbb{R})$ is a partially ordered Banach algebra with respect to above supremum norm, multiplication and the partially order relation in $C(J, \mathbb{R})$. A solution $\xi^{*}$ of the HIVP $(1.1)$ is positive if it belongs to the class of function space $C_{+}(J, \mathbb{R})$.

Theorem 3.1. Suppose that hypotheses $\left(H_{1}\right)-\left(H_{5}\right)$ and $(L S)$ hold. Then the BVP $(1.1)$ has a positive solution $x^{*}$ defined on $J$ and the sequence $\left\{x_{n}\right\}_{n=0}^{\infty}$ of successive approximations defined by

$$
\left.\begin{array}{rl}
x_{0}(t) & =u(t), \quad t \in J, \\
x_{n+1}(t) & =\left[f\left(t, x_{n}(t)\right)\right]\left(\int_{0}^{t}(t-s) g\left(s, x_{n}(t)\right) d s\right), t \in J,
\end{array}\right\}
$$

converges monotone nondecreasingly to $x^{*}$.

Proof. Set $E=C(J, \mathbb{R})$. Then, in view of Lemmas 2.4 and 2.5, $E$ is regular and every compact chain $C$ in $E$ possesses the compatibility property with respect to the norm $\|\cdot\|$ and the order relation $\preceq$ so that every compact chain $C$ is a Janhavi set in $E$.

Now by Lemma 2.2, the BVP (1.1) is equivalent to the HIE

$$
x(t)=[f(t, x(t))]\left(\int_{0}^{t}(t-s) g(s, x(t)) d s\right), t \in J .
$$

Define two operators $\mathcal{A}$ and $\mathcal{B}$ on $E$ by

$$
\mathcal{A} x(t)=f(t, x(t)), t \in J
$$

and

$$
\mathcal{B} x(t)=\int_{0}^{t}(t-s) g(s, x(t)) d s, \quad t \in J .
$$

From hypotheses $\left(\mathrm{H}_{1}\right)$ and $\left(\mathrm{H}_{3}\right)$, it follows that $\mathcal{A}$ and $\mathcal{B}$ define the operators $\mathcal{A}, \mathcal{B}: E \rightarrow K$. Now the HIE (3.4) is equivalent to the quadratic hybrid operator equation

$$
\mathcal{A} x(t) \mathcal{B} x(t)=x(t), t \in J
$$

Now, we show that the operators $\mathcal{A}$ and $\mathcal{B}$ satisfy all the conditions of Theorem 2.6 in a series of following steps.

Step I: $\mathcal{A}$ and $\mathcal{B}$ are nondecreasing operators on $E$.

Let $x, y \in E$ be such that $x \succeq y$. Then, from the hypothesis $\left(\mathrm{H}_{2}\right)$ it follows that

$$
\mathcal{A} x(t)=f(t, x(t)) \geq f(t, y(t))=\mathcal{A} x(t)
$$

for all $t \in J$. Hence $\mathcal{A} x \succeq \mathcal{A}(y)$ and that $\mathcal{A}$ is nondecreasing on $E$. Similarly, we have by hypothesis $\left(\mathrm{H}_{5}\right)$,

$$
\mathcal{B} x(t)=\int_{0}^{t}(t-s) g(s, x(s)) d \geq \int_{0}^{t}(t-s) g(s, y(s)) d s=\mathcal{B} y(t)
$$

for all $t \in J$. This implies that $\mathcal{B} x \succeq \mathcal{B} y$ whenever $x \succeq y$. Thus, $\mathcal{B}$ is also nondecreasing operator on $E$.

Step II: Next we show that $\mathcal{A}$ is partially bounded and partial $\mathcal{D}$-Lipschitz on $E$.

Now, for any $x \in E$, one has

$$
\|\mathcal{A} x\|=\sup _{t \in J}|f(t, x(t))| \leq M_{f}
$$




\section{B. C. Dhage and J. B. Dhage}

and so $\mathcal{A}$ is bounded and consequently partially bounded on $E$. Nxt let $x, y \in E$ be such that $x \succeq y$. Then, by hypotesis $\left(\mathrm{H}_{2}\right)$,

$$
|\mathcal{A} x(t)-\mathcal{A} y(t)| \leq \varphi_{f}(|x(t)-y(t)|) \leq \varphi_{f}(\|x-y\|)
$$

for all $t \in J$. Taking the supremum over $t$, we get

$$
\|\mathcal{A} x-\mathcal{A} y\| \leq \varphi_{f}(\|x-y\|)
$$

which shows that $\mathcal{A}$ is a $\mathcal{D}$-Lipschitz on $E$ with $\mathcal{D}$-function $\varphi_{f}$.

Step III: $\mathcal{B}$ is a partially contiuous and partially compact on $E$.

Let $\left\{x_{n}\right\}_{n \in \mathbb{N}}$ be a sequence in a chain $C$ such that $x_{n} \rightarrow x$ as $n \rightarrow \infty$. Since the $f$ is continuous, by dominated convergence theorem, we have

$$
\begin{aligned}
\lim _{n \rightarrow \infty} \mathcal{B} x_{n}(t) & =\lim _{n \rightarrow \infty} \int_{0}^{t}(t-s) g\left(s, x_{n}(s)\right) d s \\
& =\int_{0}^{t}(t-s)\left[\lim _{n \rightarrow \infty} g\left(s, x_{n}(s)\right)\right] d s=\mathcal{B} x(t),
\end{aligned}
$$

for all $t \in J$. This shows that $\mathcal{B} x_{n}$ converges to $\mathcal{B} x$ pointwise on $J$. Next, we show that $\left\{\mathcal{B} x_{n}\right\}_{n \in \mathbb{N}}$ is an equicontinuous sequence of functions in $E$. Now for any $t_{1}, t_{2} \in J$, one obtains

$$
\left|\mathcal{B} x_{n}\left(t_{1}\right)-\mathcal{B} x_{n}\left(t_{2}\right)\right| \leq M_{g} T\left|t_{1}-t_{2}\right|+\left|p\left(t_{1}\right)-p\left(t_{2}\right)\right| d s
$$

uniformly for all $n \in \mathbb{N}$, where $p(t)=\int_{0}^{t} M_{g}(T-s) d s$.

Since the functions $t \rightarrow|t|$ and $t \rightarrow p(t)$ is continuous on compact $J$, they are uniformly continuous there. Therefore, we have

$$
\left|p\left(t_{1}\right)-p\left(t_{2}\right)\right| \rightarrow 0 \quad \text { as } t_{1} \rightarrow t_{2}
$$

uniformly on $J$. As a result, we have that

$$
\left|\mathcal{B} x_{n}\left(t_{1}\right)-\mathcal{B} x_{n}\left(t_{2}\right)\right| \rightarrow 0 \quad \text { as } t_{1} \rightarrow t_{2},
$$

uniformly for all $n \in \mathbb{N}$. This shows that the convergence $\mathcal{B} x_{n} \rightarrow \mathcal{B} x$ is uniform and that $\mathcal{B}$ is a partially continuous operator on $E$ into itself.

Next, we show that $\mathcal{B}$ is a uniformly partially compact operator on $E$. Let $C$ be an arbitrary chain in $E$. We show that $\mathcal{B}(C)$ is uniformly bounded and equicontinuous set in $E$. First we show that $\mathcal{B}(C)$ is uniformly bounded. Let $y \in \mathcal{B}(C)$ be any element. Then there is an element $x \in C$ such that $y=\mathcal{B} x$. By hypothesis $\left(\mathrm{H}_{2}\right)$

$$
|y(t)|=|\mathcal{B} x(t)| \leq \int_{0}^{t}(t-s)|g(s, x(s))| d s \leq T^{2} M_{g}
$$

for all $t \in J$. Taking the supremum over $t$ we obtain $\|y\|=\|\mathcal{B} x\| \leq M_{g} T^{2}$ for all $y \in \mathcal{B}(C)$. Hence $\mathcal{B}(C)$ is a uniformly bounded subset of $E$. Next, proceeding with the arguments that given in Step II it can be shown that

$$
\left|y\left(t_{2}\right)-y\left(t_{1}\right)\right|=\left|\mathcal{B} x\left(t_{2}\right)-\mathcal{B} x\left(t_{1}\right)\right| \rightarrow 0 \quad \text { as } \quad t_{1} \rightarrow t_{2}
$$

uniformly for all $y \in \mathcal{B}(C)$. This shows that $\mathcal{B}(C)$ is an equicontinuous subset of $E$. Now, $\mathcal{B}(C)$ is a uniformly bounded and equicontinuous subset of functions in $E$ and hence it is compact in view of Arzelá-Ascoli theorem. Consequently $\mathcal{B}$ is a uniformly partially compact operator on $E$ into itself.

Step IV: $\mathcal{A}$ and $\mathcal{B}$ satisfy the growth inequality $M_{B} \varphi_{A}(r)<r, r>0$. 
Approximation results for nonlinear hybrid boundary value problems

Now, it can be shown $\|\mathcal{B}(C)\| \leq T^{2} M_{g}=M_{\mathcal{B}}$ for all chain $C$ in $E$. Therefore, we obtain

$$
M_{\mathcal{B}} \varphi_{\mathcal{A}}(r)=T^{2} M_{g} \varphi_{f}(r)<r
$$

for all $r>0$ and so the hypothesis (c) of Theorem 2.6 is satisfied.

Step VI: The function $u$ satisfies the operator inequality $u \preceq \mathcal{A} u \mathcal{B} u$.

By hypothesis (LS), the HIVP (1.1) has a lower solution $u$ defined on $J$. Then, we have

$$
\left.\begin{array}{c}
\frac{d^{2}}{d t^{2}}\left(\frac{u(t)}{f(t, u(t))}\right) \leq g(t, u(t)) \text { a.e. } t \in J, \\
\frac{u(0)}{f(0, u(0))}=0,\left.\quad\left(\frac{u(t)}{f(t, u(t))}\right)^{\prime}\right|_{t=0}=0,
\end{array}\right\}
$$

By using this, the fundamental theorem of calculus and the definitions of the operators $\mathcal{A}$ and $\mathcal{B}$, it can be shown that the function $u \in C(J, \mathbb{R})$ satisfies the relation $u \preceq \mathcal{A} u \mathcal{B} u$ on $J$.

Thus, $\mathcal{A}$ and $\mathcal{B}$ satisfy all the conditions of Theorem 2.6 and so the quadratic hybrid operator equation $\mathcal{A} x \mathcal{B} x=x$ has a positive solution $x^{*}$ and the sequence $\left\{x_{n}\right\}_{n=0}^{\infty}$ of successive iterations defined by $x_{n+1}=$ $\mathcal{A} x_{n} \mathcal{B} x_{n}$ with initial term $x_{0}=u$ converges monotone nondecreasingly to $x^{*}$. Therefore, the HIE (3.4) and consequently the HIVP (1.1) has a positive solution $x^{*}$ and the sequence $\left\{x_{n}\right\}_{n=0}^{\infty}$ of successive approximations defined by (3.3) with $x_{0}=u$, converges monotone nondecreasingly to $x^{*}$. This completes the proof.

Remark 3.2. The conclusion of Theorem 3.1 also remains true if we replace the hypothesis (LS) with (US). The proof of Theorem 3.1 under this new hypothesis is similar and can be obtained by closely observing the same arguments with appropriate modifications. In this case the sequence $\left\{x_{n}\right\}_{n=0}^{\infty}$ defined by (3.3) with $x_{0}(t)=$ $v(t), \quad t \in[0, T]$, converges montone nonincrasingly to the solution $x^{*}$ of he HIVP (1.1) on $J$. Again, the existence and approximation result, Theorem 3.1 includes similar result for the positive solution of the HIVP (1.2) as a special case.

Remark 3.3. We note that if the HIVP (1.1) has a lower solution $u \in A C^{1}(J, \mathbb{R})$ as well as an upper solution $v \in A C^{1}(J, \mathbb{R})$ such that $u \preceq v$, then under the given conditions of Theorem 3.1 it has corresponding solutions $x_{*}$ and $y^{*}$ and these solutions satisfy the inequality

$$
u=x_{0} \preceq x_{1} \preceq \cdots \preceq x_{n} \preceq x_{*} \preceq y^{*} \preceq y_{n} \preceq \cdots \preceq y_{1} \preceq y_{0}=v .
$$

Hence $x_{*}$ and $y^{*}$ are respectively the minimal and maximal impulsive solutions of the HIVP (1.1) in the vector segment $[u, v]$ of the Banach space $E=C(J, \mathbb{R})$, where the vector segment $[u, v]$ is a set of elements in $C(J, \mathbb{R})$ defined by

$$
[u, v]=\{x \in C(J, \mathbb{R}) \mid u \preceq x \preceq v\} .
$$

This is because of the order cone $K$ defined by (3.2) is a closed convex subset of $C(J, \mathbb{R})$. However, we have not used any property of the cone $K$ in the main existence results of this paper. A few details concerning the order relation by the order cones and the Janhavi sets in an ordered Banach space are given in Dhage [4].

\section{An Example}

\section{Example 4.1.}

Given a closed interval $J=[0,1]$ in $\mathbb{R}$, consider the nonlinear HIVP of hybrid differential equations

$$
\left.\begin{array}{c}
\frac{d^{2}}{d t^{2}}\left(\frac{x(t)}{f(t, x(t))}\right)=\tanh x(t)+1 \text { a.e. } t \in J, \\
x(0)=0, \quad x^{\prime}(0)=0,
\end{array}\right\}
$$


where the function $f: J \times \mathbb{R} \rightarrow \mathbb{R} \backslash\{0\}$ is defined by

$$
f(t, x)=\left\{\begin{array}{cl}
1, & \text { if } x \leq 0 \\
1+\frac{x}{1+x}, & \text { if } x>0
\end{array}\right.
$$

Then the function $f$ satisfies the hypotheses $\left(\mathrm{H}_{1}\right)-\left(\mathrm{H}_{2}\right)$ with $M_{f}=2$ and $\varphi_{f}(r)=\frac{r}{1+\xi^{2}}, 0 \leq \xi \leq r$. Here $g(t, x)=\tanh x+1$ and satisfies the hypotheses $\left(\mathrm{H}_{3}\right)-\left(\mathrm{H}_{5}\right)$ with $M_{g}=2$. Now the HIVP (4.1) is equivalent to the HIE

$$
x(t)=[f(t, x(t))]\left(\int_{0}^{t}(t-s)[\tanh x(s)+1] d s\right), t \in[-1,1],
$$

It can be verified that the function $u \in C(J, \mathbb{R})$ defined by $u(t)=-t^{2}$ and $v(t)=4 t^{2}$ are respectively the lower and upper solutions of the HIVP (4.1) on [0,1]. Hence, by an application of Theorem 3.1, the HIVP (4.1) has a positive solution $x^{*}$ and the sequence $\left\{x_{n}\right\}_{n=0}^{\infty}$ of successive approximations defined by

$$
\begin{aligned}
x_{0}(t) & =-t^{2}, \quad t \in[0,1], \\
x_{n+1}(t) & =\left[f\left(t, x_{n}(t)\right)\right]\left(\int_{0}^{t}(t-s)\left[\tanh x_{n}(s)+1\right] d s\right), t \in[0,1],
\end{aligned}
$$

converges monotone nondecreasingly to $x^{*}$. Similarly, by Remark 3.2, the sequence $\left\{y_{n}\right\}_{n=0}^{\infty}$ of successive approximations defined by

$$
\begin{aligned}
y_{0}(t) & =4 t^{2}, t \in[0,1], \\
y_{n+1}(t) & =\left[f\left(t, y_{n}(t)\right)\right]\left(\int_{0}^{t}(t-s)\left[\tanh y_{n}(s)+1\right] d s\right), t \in[0,1],
\end{aligned}
$$

converges monotone non-increasingly to the positive solution $y^{*}$ of the HIVP $(4.1)$ on $[0,1]$.

\section{References}

[1] K. Deimling, Nonlinear Functional Analysis, Springer Verlag, 1985.

[2] B.C. Dhage, Nonlinear functional boundary value problems involving Carathèodory, Kyungpook Mathematical Journal, 46(2006), 421-441.

[3] B.C. Dhage, Partially condensing mappings in partially ordered normed linear spaces and applications to functional integral equations, Tamkang Journal of Mathematics, 45(4)(2014), 397-427.

[4] B.C. Dhage, Coupled and mixed coupled hybrid fixed point principles in a partially ordered Banach algebra and PBVPs of nonlinear coupled quadratic differential equations, Differ. Equ. Appl., 11(1)(2019), 1-85.

[5] B.C. Dhage, S.B. Dhage, Approximating solutions of nonlinear second order ordinary differetial equations, Malaya Journal of Matematik, 4(1)(2016), 8-18.

[6] B.C. Dhage, S.B. Dhage, Approximating soltions of PBVPs of second order ordinary differetial equations via hybrid fixed point theory, Electronic J. Diff. Equations, 20(1)(2015), 1-10. 
Approximation results for nonlinear hybrid boundary value problems

[7] B. C. Dhage, M. Imdad, Asymptotic behaviour of nonlinear quadratic functional integral equations involving Carathèodory, Nonlinear Analysis 71 (2009), 1285-1291.

[8] A. Granas, Fixed Point Theory, Springer Verlag 2003.

This is an open access article distributed under the Creative Commons Attribution (c) License, which permits unrestricted use, distribution, and reproduction in any medium, provided the original work is properly cited. 